\title{
OPEN Novel statistical approach for assessing the persistence of the circadian rhythms of social activity from telephone call detail records in older adults
}

\author{
Timothée Aubourg ${ }^{1,2,3 凶}$, Jacques Demongeot ${ }^{1,3,4}$ \& Nicolas Vuillerme ${ }^{1,3,4}$
}

How circadian rhythms of activity manifest themselves in social life of humans remains one of the most intriguing questions in chronobiology and a major issue for personalized medicine. Over the past years, substantial advances have been made in understanding the personal nature and the robustness-i.e. the persistence-of the circadian rhythms of social activity by the analysis of phone use. At this stage however, the consistency of such advances as their statistical validity remains unclear. The present paper has been specifically designed to address this issue. To this end, we propose a novel statistical procedure for the measurement of the circadian rhythms of social activity which is particularly well-suited for the existing framework of persistence analysis. Furthermore, we illustrate how this procedure works concretely by assessing the persistence of the circadian rhythms of telephone call activity from a 12-month call detail records (CDRs) dataset of adults over than 65 years. The results show the ability of our approach for assessing persistence with a statistical significance. In the field of CDRs analysis, this novel statistical approach can be used for completing the existing methods used to analyze the persistence of the circadian rhythms of a social nature. More importantly, it provides an opportunity to open up the analysis of CDRs for various domains of application in personalized medicine requiring access to statistical significance such as health care monitoring.

Circadian rhythms are endogenous processes characterized by a period close to $24 \mathrm{~h}$ depending on individuals ${ }^{1}$. Their ubiquity makes them one of the most perceptible phenomena in an individual's life, reflecting and affecting all his fundamental domains of activity: biological, physical and social ${ }^{2-4}$. In the field of health care monitoring, the analysis of the biological, physical and social mechanisms involved in the emergence, maintaining and characterization of the circadian rhythms does represent an increasingly important issue. In medicine, this importance was evidenced by the recent awarding of Nobel Prize for Medicine in 2017 to Michael Young, Michael Rosbash and Jeffrey Hall for their discoveries into the molecular mechanisms controlling the circadian rhythms ${ }^{5}$. From a clinical perspective, it is now well recognized that the deep comprehension of the circadian rhythms represents an opportunity for better managing a patient's health in time ${ }^{6}$. In particular, for the clinical practice, this comprehension can help the health professional properly address his patients' needs and care by delivering the adequate treatment at the optimal time of day ${ }^{7}$. For several decades now, such an interest for the analysis of the circadian rhythms of activity has been present in the ever-growing literature on subjects related to their biological and physical manifestations and on their association with health outcomes (see ${ }^{8-10}$ for recent reviews).

Nowadays, the digital ubiquity characterizing our 'hyper-connected' society brings new paradigms for addressing the understanding of circadian rhythms in health. In particular, while the study of the biological and physical mechanisms controlling the circadian rhythms is well addressed yet by, respectively, the fields of chronobiology and that of actigraphy, a new ubiquitous computing paradigm has emerged for addressing their social manifestations. This paradigm sets on the observation that modern technologies, and phone technologies particularly, are now completely disseminated inside our daily social lives ${ }^{11}$. Accordingly, the analysis of their

${ }^{1}$ Univ. Grenoble Alpes, AGEIS, Grenoble, France. ${ }^{2}$ Orange Labs, Meylan, France. ${ }^{3}$ LabCom Telecom4Health, Univ. Grenoble Alpes \& Orange Labs, Grenoble, France. ${ }^{4}$ Institut Universitaire de France, Paris, France. ${ }^{\square}$ email: timothee.aubourg@gmail.com 
generated data could help to better model and understand the social aspects of an individual's behavior at a daily scale ${ }^{11}$. From a clinical perspective, this approach is of strong interest given that the social manifestations of circadian rhythms are, evidently, not easily perceptible for the biological and physical approaches currently used in chronobiology and actigraphy.

Along these lines, a recent body of literature has emerged around the use of phone technologies for social and behavioral modeling ${ }^{12-23}$. On the whole, this literature emphasizes the relevance of call detail records (CDRs)which synthetize telephone calls and SMS exchanges of a telephone user-for the analysis of the circadian rhythms of social interactions that occur at telephone ${ }^{15}$. In particular, it is evinced that CDRs analysis permit to investigate continuously, objectively and unobtrusively, essential properties of such social rhythms ${ }^{24-28}$. Following this train of thought, recent works have reported how the persistence in time of social interactions occurring during the day at telephone could possibly be one of these properties ${ }^{24-27}$. For a given individual, persistence is considered as the maintaining in time of the robustness and distinctiveness of a measured phenomenon at telephone, also named pattern, against a comparative population ${ }^{21}$. A measured phenomenon that is characterized as persistent for an individual is then considered as a signature of his telephone activity ${ }^{21}$.

The first persistence analysis applied to telephone call activity was carried out by Saramäki et al. from the Aalto University in Finland in $2014^{21}$. In their PNAS article, these authors reported on an 18-month CDRs dataset of 30 students the existence of social signatures in the way students allocate their volume of communications with their social network over successive months. In short, each student was found to present a pattern of communicating with his social network which varies from one to another. And this pattern was found to be maintained in time despite the occurrence of a major social turn-over induced by high-school-to-university or high-school-towork transitions. Following the methodology described by Saramäki et al., Alessandretti et al. reported on 850 high resolution trajectories and call detail records of participants in a 24 months longitudinal experiment, the Copenhagen Networks Study (CNS) ${ }^{29}$, the existence of social and spatial signatures over months ${ }^{30}$. In particular, they show how CDRs analysis permits to evidence persistence in the way an individual exploits known assets in the social and spatial spheres. Finally, for the circadian rhythms, Aledavood et al. reported on the same dataset as that used $\mathrm{in}^{21}$ the existence of temporal signatures in the way participants distribute their frequency of outgoing calls according to the hour of day ${ }^{25}$. Other studies further show how these persistent circadian patterns can be also evidenced with other types of social interactions ${ }^{26,27}$, such as text messages ${ }^{26}$, or with other populations as the older one ${ }^{24,28}$.

In the field of health care monitoring, such results on the robustness and distinctiveness of the digital signatures of telephone activity can permit to better understand the social elements involved in the circadian rhythms of activity that are of a social and personal nature. These elements can be used for improving health care monitoring by informing health professionals on the structure and quality of a patient's daily rhythm of his social activity at telephone. In the research community, such an interest in the analysis of the daily rhythms of telephone activity is well highlighted by recent studies on this topic ${ }^{24,28,31-34}$ and has contributed to the emergence and success of new promising innovative fields in health, as reflected by the digital phenotyping one $e^{35}$, the field of digital psychiatry ${ }^{36}$ or that of mobile health $(m H e a l t h)^{13}$ just for naming the most cited ones (see the work shared in the Lancet journal by a recent commission on the future of psychiatry for instance ${ }^{36}$ ).

At this stage however, concerning the persistent nature of the digital activity measured from phone technologies, it must be said that the statistical validity of these promising results remains unclear. In $^{21}$ for instance, the analytical methodology used for assessing the robustness and distinctiveness of individual patterns of telephone call activity involves mean estimators only and no statistical validation. Similar estimators were used in ${ }^{30}$, in conjunction with the following decision rule: given a phenomenon observed at telephone for an individual $i$ from a population $A=\{j, j \in[1 \ldots, i, \ldots, M])$, if its distinctiveness over time is validated "for most $\mathrm{j}$ [with $j \neq i$ ], we can conclude that for individual $i$, fluctuations of the [measured phenomenon] are negligible compared to the difference with other individuals". This method is exactly the same as that used for assessing the persistent nature of the circadian rhythms of telephone call activity, which is reported in ${ }^{24-27}$.

For the clinical practice, assessing the statistical significance of the persistent nature of the circadian rhythms of telephone call activity is essential before validating their consistency. Thus, at this stage, whether and how the existing results reported in the literature on the subject could be validated statistically and within an appropriate methodology remains to be established. The present paper has been specifically designed to address this issue. To this end, we introduce a novel statistical approach for measuring the persistence of the circadian rhythms of activity at telephone which is particularly well-suited for the existing framework of persistence analysis ${ }^{21}$. Then, we illustrate how this procedure works concretely by assessing the persistence of the circadian rhythms of telephone call activity on a 12-month CDRs dataset of adults over than 65 years. Three cases are tested separately: (1) outgoing, (2) incoming, and (3) total call activities. The results are discussed and, at the end, a future direction is proposed.

\section{Methods}

Study population and data collection. This study is based on 12 months of CDRs for 26 volunteers (20 women, 6 men; median age: 84 years; range: 71-91 years). CDRs provided by the local communication service provider were collected from the personal telephone(s) of the volunteers. Each CDRs contains the date, hour, source ID, recipient ID, direction, and duration of call (in seconds). Note that the telephone owners and the telephone contacts remained anonymous. The present study and its corresponding experimental protocols were declared to the French Data Protection Authority (CNIL registered data protection officer, France Telecom 2011 $\mathrm{n}^{\circ} 44$ ). All experimental methods were carried out as per the relevant regulations and written informed consent was obtained from all participants before data were collected and anonymized. This study is secondary analysis of previous publised and unpublished data studies ${ }^{28,37}$. 


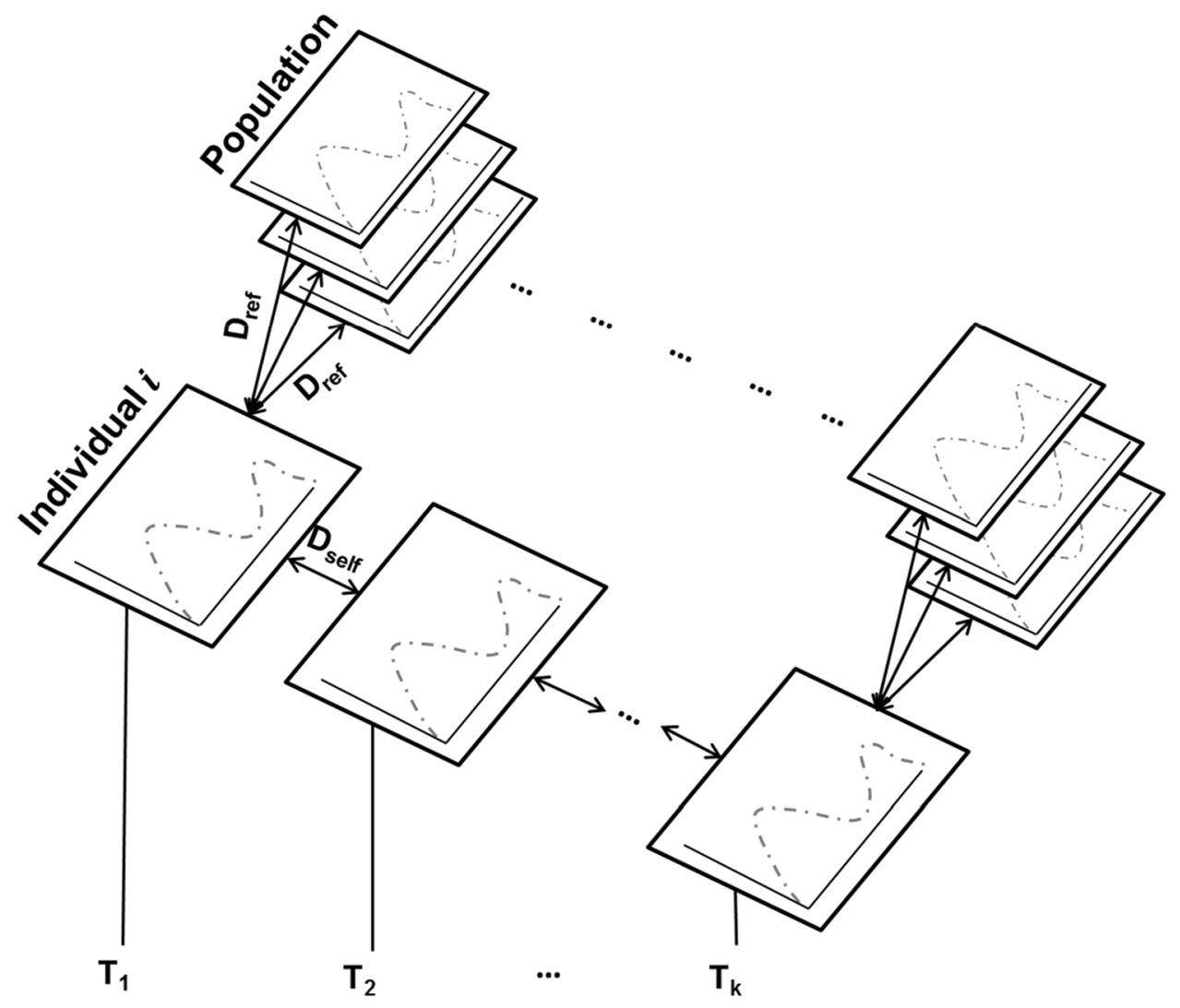

Figure 1. Illustrative view of the persistence analysis process applied to the circadian rhythms of activity. Here, $\mathrm{D}_{\text {self }}$ corresponds to the dissimilarity measured between the daily rhythms of two successive periods of time (which vary from $\mathrm{T}_{1}$ to $\mathrm{T}_{\mathrm{k}}$ ) of an individual $i$, named intra-individual dissimilarity. $\mathrm{D}_{\text {ref }}$ correspond to the dissimilarities measured between the daily rhythms of an individual $i$ and the other individuals of the observed population within a same time period, named inter-individual dissimilarities. Persistence is validated if and only if $D_{\text {self }}$ that is measured between two successive time windows tends to minimize $D_{\text {ref }}$ 's that are measured for each of these two time windows separately.

Data analysis. The statistical procedure used for assessing the persistence of the circadian rhythms of telephone call activity relies on a method originally proposed by Saramäki et al. in their PNAS article ${ }^{21}$, and which is illustrated on Fig. 1.

Here, we propose both to formalize this existing method into a broader analytical procedure that introduces supplementary steps for ensuring the statistical validation of results (step 5.1, step 5.2 and step 5.3). Furthermore, this procedure distinguishes two cases of analysis: (1) one that compares two successive temporal windows, and (2) another that extends case (1) for comparing more than two successive temporal windows.

Statistical procedure. Persistence analysis for two successive time periods $\left(N_{T}=2\right)$. Let a given population of $n$ individuals $A=\{i, i \in[1, \ldots, n]\}$ observed over two successive periods of time $\mathrm{T}_{1}$ and $\mathrm{T}_{2}$. The persistence analysis consists of the five following steps:

Step 1: Time discretization The individuals' CDRs are coarse-grained into two successive periods of time, $\mathrm{T}_{1}$ and $\mathrm{T}_{2}$. Each of these two periods is divided into 24 one-hour time slots.

Step 2: Calculation of daily rhythm For each individual $i$ from population $A$, the daily rhythm of telephone calls is calculated for each period $\mathrm{T}_{1}$ and $\mathrm{T}_{2}$ by using the function $f_{i}(t)=\frac{n_{i}(t)}{\sum_{t=0}^{23} n_{i}(t)}$, where $n_{i}(t)$ is the number of calls in time slot $t$ of individual $i$, with $t \in[0 \ldots 23]$.

Step 3: Intra-individual dissimilarity We denote by $D_{\text {self }}$ the dissimilarity measure of the individual's daily rhythms between $\mathrm{T}_{1}$ and $\mathrm{T}_{2}$. $D_{\text {self }}$ is given by:

$$
D_{\text {self }}\left(i, \mathrm{~T}_{1}, T_{2}\right)=\sqrt{\mathrm{D}\left(i, P_{i}^{\mathrm{T}_{1}}, P_{i}^{\mathrm{T}_{2}}\right)}
$$

where $\mathrm{D}$ is a dissimilarity measure, $P_{i}^{\mathrm{T}_{1}}\left(P_{i}^{\mathrm{T}_{2}}\right)$ is the discrete probability distribution of the call fractions for individual $i$ calculated at time period $\mathrm{T}_{1}\left(\mathrm{~T}_{2}\right)$, in step 2 .

Finally, we denote $y_{i}=D_{\text {self }}\left(i, \mathrm{~T}_{1}, \mathrm{~T}_{2}\right)$.

Step 4: Inter-individual dissimilarity. We denote by $D_{\text {ref }}$ a dissimilarity measure between two daily rhythms for two distinct individuals in the same time period. $D_{\text {ref }}$ is given by: 


$$
D_{\mathrm{ref}}\left(i, j, \mathrm{~T}_{\mathrm{k}}\right)=\sqrt{\mathrm{D}\left(P_{i}^{\mathrm{T}_{\mathrm{k}}}, P_{j}^{\mathrm{T}_{\mathrm{k}}}\right)}
$$

where $\mathrm{D}$ is a dissimilarity measure, $P_{i}^{\mathrm{T}_{\mathrm{k}}}\left(P_{j}^{\mathrm{T}_{\mathrm{k}}}\right)$ is the discrete probability distribution of call fractions for individual $i(j)$ at time period $\mathrm{T}_{\mathrm{k}}$, with $i, j \in[1 . . n], i \neq j$, and $\mathrm{k} \in\{1,2\}$.

In the following, we denote by $x_{i}^{\mathrm{T}_{1}}=\left(x_{i, j}^{\mathrm{T}_{1}}\right)_{j=1, n ; i \neq j}$ a sample of observations of size $\mathrm{n}-1$ corresponding to the inter-individual dissimilarities calculated between a given individual $i$ and each of the other individuals $j$ from population $A$, such as $i \neq j$, within period $\mathrm{T}_{1}$.

Similarly, we denote by $x_{i}^{\mathrm{T}_{2}}=\left(x_{i, j}^{\mathrm{T}_{2}}\right)_{j=1, n ; i \neq j}$ a sample of observation of size $\mathrm{n}-1$ corresponding to the interindividual dissimilarities calculated between a given individual $i$ and each of the other individuals $j$ from the population $A$, and such as $i \neq j$, within period $\mathrm{T}_{2}$.

Step 5: Persistence assessment. Persistence is assessed by comparing how the intra-individual dissimilarity of a given individual's daily rhythm between $\mathrm{T}_{1}$ and $\mathrm{T}_{2}$ lies in comparison with the values of his inter-individual dissimilarities in each of the two time periods $\mathrm{T}_{1}$ and $\mathrm{T}_{2}$. A daily rhythm of telephone call activity is found persistent if and only if the intra-individual dissimilarity tends to minimize the set of inter-individual dissimilarities. For an individual $i$, the assessment consists of a sign test of quantile as follows:

Step 5.1 First, We set $z_{i}=\left(z_{i, j}^{\mathrm{T}_{\mathrm{k}}}\right)_{j=1, n ; i \neq j ; k=1,2}=\left(1_{\left\{y_{\mathrm{i}}-x_{i, j}^{\mathrm{T}_{\mathrm{k}}}>0\right\}}\right)_{j=1, n ; i \neq j ; k=1,2}$, the vector resulting of the comparison of each inter-individuals dissimilarity $x_{i}^{\mathrm{T}_{\mathrm{k}}}$ in each period $\mathrm{T}_{\mathrm{k}}$ with the individual $i$ 's intra-dissimilarity $y_{i}$, where the dimension of the vector $x_{i}^{\mathrm{T}_{\mathrm{k}}}$ is $\mathrm{n}-1$ and $\mathrm{k}=\{1,2\}$, which corresponds to $2 n-2$ comparisons. Each component of $z_{i}$ has values in $\{0,+1\}$, where +1 corresponds to a success for obtaining an inter-individual dissimilarity lower than an intra-individual dissimilarity, and 0 to a failure.

Step 5.2 Let set $\mathrm{N}_{i}^{+}=\Sigma_{j=1, n ; i \neq j, k=1,2} z_{i}^{T_{\mathrm{k}}}$ being the total number of successes of individual $i$ in the two periods $\mathrm{T}_{1}$ and $\mathrm{T}_{2}$.

Step 5.3 We set the null hypothesis $\mathrm{H}_{0}=$ \{the probability for obtaining an inter-individual dissimilarity lower than an intra-individual dissimilarity is equal to $q\}$, with $q$ set at $1 / 2$ by default (case of median test).

Hence, under $\mathrm{H}_{0}$, each of the observed inter-individual dissimilarities has a probability $q$ for being lower than the intra-individual dissimilarity $y_{i}$. We thus have $\mathrm{N}_{\mathrm{i}}^{+} \sim B(2 n-2, q)$, if the behavior of individuals $j$ in period $\mathrm{T}_{1}$ and $\mathrm{T}_{2}$ can be considered as independent.

In this context, considering population $\mathrm{A}$, and time periods $\mathrm{T}_{1}$ and $\mathrm{T}_{2}$, a significant $\mathrm{P}$-value obtained from a binomial test brings a statistical element of response that supports the persistence of the daily rhythm of telephone call activity of individual $i$.

Persistence analysis for more than two successive time periods $\left(N_{T}>2\right)$. Let a given population of $n$ individuals $A=\{i, i \in[1 \ldots n]\}$ observed over $\mathrm{N}_{\mathrm{T}}$ successive periods of time. In this context, for a given individual $i \in A$, we evaluate his trend for having a persistent behavior at telephone between successive time periods.

To this end, we run the same persistence analysis than that described above for each two successive periods of time. We consider two types of events from the obtained results: (1) "Persistence" that corresponds to a significant p-value, and (2) "No Persistence" that corresponds to a non-significant $P$-value.

We consider the sample of observations $v$ of length $\mathrm{N}_{\mathrm{T}}-1$, where each element of $v$ is at value in $\{0,1\}$ with 1 (resp. 0) corresponding to a "Persistence" event (resp. "No persistence") obtained from the comparisons. Finally, a sign test is applied to $v$. A significant result indicates individual $i$ tends to have a persistent behavior at telephone with regards to population $A$ and the $\mathrm{N}_{\mathrm{T}}$ successive periods of time.

In practice, we used $P$-value $<0.05$ in statistical tests as the level of significance. All statistical calculations were done in the R software environment (version 3.1.6; R Foundation for Statistical Computing, Vienna, Austria).

Approval of the experimental protocol. The present study and its corresponding experimental protocols were approved by the French Data Protection Authority (CNIL registered data protection officer, France Telecom $2011 \mathrm{n}^{\circ} 44$ ). All experimental methods were carried out as per the relevant regulations and written informed consent was obtained from all participants before data were collected and anonymized.

\section{Results}

We applied the analytical procedure proposed in the Methods section on a 12-month CDRs dataset of adults over 65 years. Three cases are tested separately: (1) outgoing, (2) incoming, and (3) total call activities. We considered a two successive temporal window $\left(\mathrm{N}_{\mathrm{T}}=2\right)$, and we used the Jenson-Shannon Divergence dissimilarity (denoted $\mathrm{D}$ in Methods section) as dissimilarity measurement.

Figure 2 presents for each individual the two daily rhythms of outgoing telephone call activity for the two successive time periods of 6 months each, $T_{1}$ and $T_{2}$. The differences between $T_{1}$ and $T_{2}$ are illustrated by a colored area differentiating the two corresponding curves: (1) in green when the ratio of calls corresponding to $\mathrm{T}_{1}$ is higher than $\mathrm{T}_{2}$, and (2) in red for the opposite. Figure 2 illustrates the similarities and differences observed between two successive rhythms of telephone call activity of the same individual. On this figure, we can observe that some of them, such as individuals A or B for instance, seem to exhibit a few differences between $\mathrm{T}_{1}$ and $\mathrm{T}_{2}$. Others, such as individuals $\mathrm{W}$ or $\mathrm{Y}$ for instance, seem to exhibit more pronounced differences. 


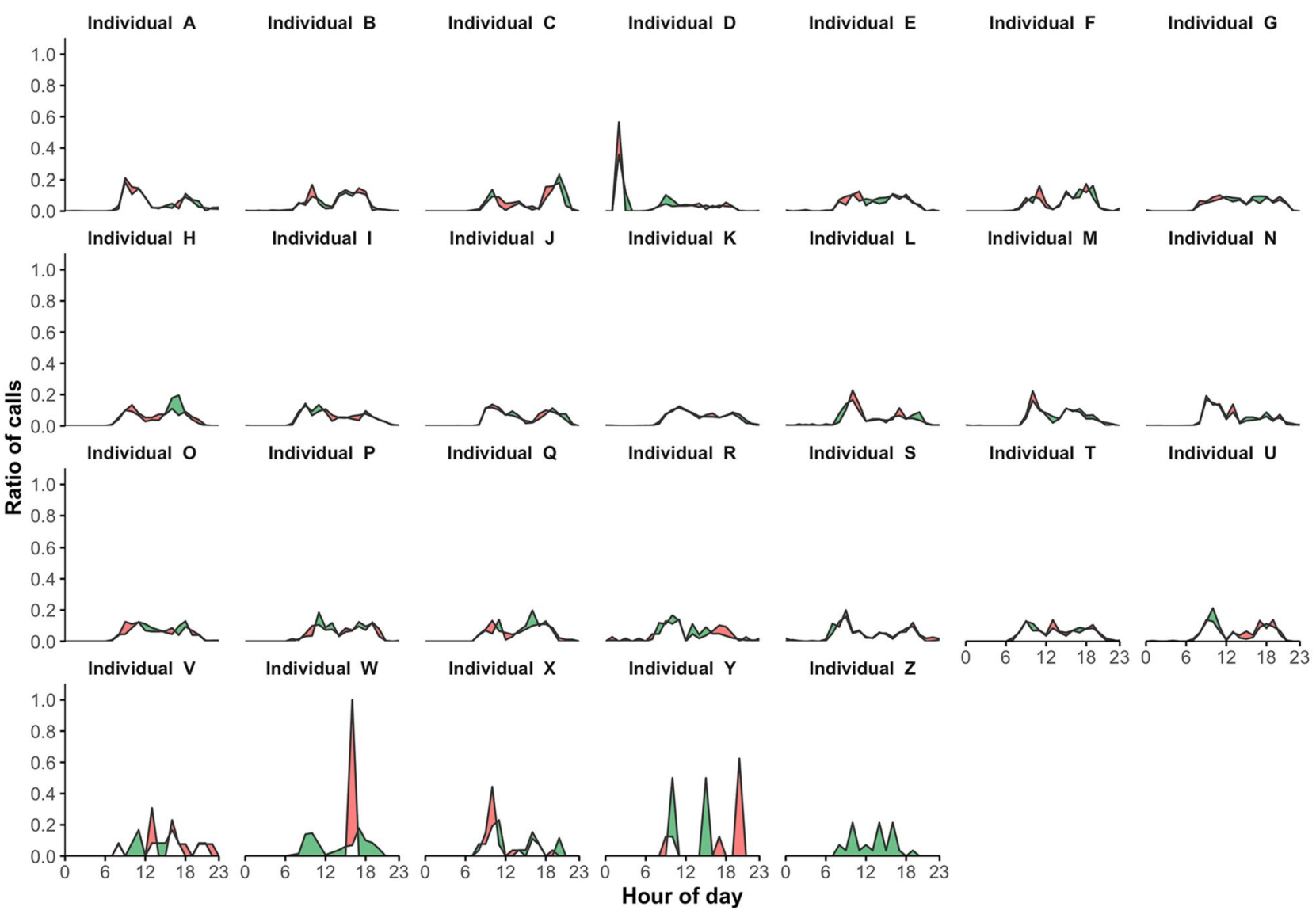

Figure 2. Comparison of two daily rhythms of outgoing telephone call activity. Daily rhythms of outgoing telephone call activity are calculated for the two successive time periods of 6 months each, $\mathrm{T}_{1}$ and $\mathrm{T}_{2}$. Their differences are illustrated by coloring the area between the two corresponding curves: (1) in green when the ratio of calls corresponding to $\mathrm{T}_{1}$ is higher than $\mathrm{T}_{2}$, and (2) in red for the opposite.

The statistical results are stored in Table 1. For a given individual $i, \mathrm{~N}_{\mathrm{i}}^{+}$informs on the number of his interdissimilarities lower than his intra-dissimilarity, whereas the corresponding p-value permit to associate $\mathrm{N}_{\mathrm{i}}^{+}$with a persistent or not persistent nature that is statistically valid. For the present dataset, the statistical results stored in Table 1 show that individuals V, W, Y, Z do not present a circadian rhythm of telephone call activity that is significantly persistent ( $\mathrm{p}$-values $>0.05$ ) against the population observed. On the contrary, the other individuals show a significant persistence $(\mathrm{P}$-values $<0.05)$.

Figure 3 presents for each individual the two daily rhythms of his incoming telephone calls for the two successive time periods of 6 months each, $\mathrm{T}_{1}$ and $\mathrm{T}_{2}$. Again, the statistical results are stored in Table 1. It appears that $\mathrm{W}$ and $\mathrm{Z}$ are the only ones who do not show significant persistence (P-values $<0.05$ ). For individuals $\mathrm{V}$ and $\mathrm{Y}$, this implies that the persistent nature of their circadian rhythms of telephone call activity depends on the nature of the direction of calls considered.

Figure 4 presents for each individual the two daily rhythms of his total telephone call activity for the two successive time periods of 6 months each, $T_{1}$ and $T_{2}$. The statistical results are stored in Table 1. Again, $W$ and $\mathrm{Z}$ are the only ones who do not show significant persistence (P-values $<0.05)$.

\section{Discussion}

The present study has been carried out for addressing the absence of statistical consistency in the current methods of persistence analysis applied to the circadian rhythms of telephone activity. To this end, we show that the methods currently used in the literature can be reformulated into a well-defined and simple non-parametrical statistical problem. In this train of thought, we propose a novel statistical approach that permits to measure the circadian rhythms of telephone activity using a sign test of quantiles and which permits to ensure the results' statistical validity. Then, we illustrate an application of this statistical procedure by assessing the persistence of the circadian rhythms of telephone call activity on a 12-month CDRs dataset of adults over 65 years. Three cases are tested separately: (1) outgoing, (2) incoming, and (3) total call activities. On the whole, the results show the ability of our approach for assessing persistence with a statistical significance. In particular, it permits to figure out that, in this CDRs dataset, even if most of the older individuals from the observed population show a significant persistent circadian behavior at telephone, this observation (1) does not stand systematically and 


\begin{tabular}{|c|c|c|c|c|c|c|c|c|c|}
\hline \multirow[b]{2}{*}{ Individual } & \multicolumn{3}{|c|}{ Outgoing calls } & \multicolumn{3}{|c|}{ Incoming calls } & \multicolumn{3}{|l|}{ Total calls } \\
\hline & P-value & $\mathbf{N}_{+}$ & $\mathbf{N}_{\text {comp }}$ & P-value & $\mathbf{N}_{+}$ & $\mathbf{N}_{\text {comp }}$ & P-value & $\mathbf{N}_{+}$ & $\mathbf{N}_{\text {comp }}$ \\
\hline A & $8.88 \mathrm{E}-16$ & 0 & 50 & $8.88 \mathrm{E}-16$ & 0 & 50 & $8.88 \mathrm{E}-16$ & 0 & 50 \\
\hline B & $8.88 \mathrm{E}-16$ & 0 & 50 & $8.88 \mathrm{E}-16$ & 0 & 50 & $8.88 \mathrm{E}-16$ & 0 & 50 \\
\hline $\mathrm{C}$ & $4.53 \mathrm{E}-14$ & 1 & 50 & $8.88 \mathrm{E}-16$ & 0 & 50 & $8.88 \mathrm{E}-16$ & 0 & 50 \\
\hline $\mathrm{D}$ & $8.88 \mathrm{E}-16$ & 0 & 50 & $8.88 \mathrm{E}-16$ & 0 & 50 & $8.88 \mathrm{E}-16$ & 0 & 50 \\
\hline $\mathrm{E}$ & $2.23 \mathrm{E}-10$ & 4 & 50 & $2.81 \mathrm{E}-06$ & 9 & 50 & $2.81 \mathrm{E}-06$ & 9 & 50 \\
\hline $\mathrm{F}$ & $8.88 \mathrm{E}-16$ & 0 & 50 & $4.53 \mathrm{E}-14$ & 1 & 50 & $8.88 \mathrm{E}-16$ & 0 & 50 \\
\hline G & $8.88 \mathrm{E}-16$ & 0 & 50 & $8.88 \mathrm{E}-16$ & 0 & 50 & $8.88 \mathrm{E}-16$ & 0 & 50 \\
\hline $\mathrm{H}$ & $5.82 \mathrm{E}-07$ & 8 & 50 & $8.88 \mathrm{E}-16$ & 0 & 50 & $2.23 \mathrm{E}-10$ & 4 & 50 \\
\hline I & $8.88 \mathrm{E}-16$ & 0 & 50 & $8.88 \mathrm{E}-16$ & 0 & 50 & $8.88 \mathrm{E}-16$ & 0 & 50 \\
\hline $\mathrm{J}$ & $8.88 \mathrm{E}-16$ & 0 & 50 & $8.88 \mathrm{E}-16$ & 0 & 50 & $8.88 \mathrm{E}-16$ & 0 & 50 \\
\hline $\mathrm{K}$ & $8.88 \mathrm{E}-16$ & 0 & 50 & $1.30 \mathrm{E}-03$ & 14 & 50 & $8.88 \mathrm{E}-16$ & 0 & 50 \\
\hline $\mathrm{L}$ & $4.53 \mathrm{E}-14$ & 1 & 50 & $8.88 \mathrm{E}-16$ & 0 & 50 & $8.88 \mathrm{E}-16$ & 0 & 50 \\
\hline $\mathrm{M}$ & $8.88 \mathrm{E}-16$ & 0 & 50 & $8.88 \mathrm{E}-16$ & 0 & 50 & $8.88 \mathrm{E}-16$ & 0 & 50 \\
\hline $\mathrm{N}$ & $8.88 \mathrm{E}-16$ & 0 & 50 & $8.88 \mathrm{E}-16$ & 0 & 50 & $8.88 \mathrm{E}-16$ & 0 & 50 \\
\hline $\mathrm{O}$ & $5.82 \mathrm{E}-07$ & 8 & 50 & $8.88 \mathrm{E}-16$ & 0 & 50 & $4.53 \mathrm{E}-14$ & 1 & 50 \\
\hline $\mathrm{P}$ & $1.13 \mathrm{E}-12$ & 2 & 50 & $3.25 \mathrm{E}-02$ & 18 & 50 & $4.51 \mathrm{E}-05$ & 11 & 50 \\
\hline Q & $1.30 \mathrm{E}-03$ & 14 & 50 & $4.53 \mathrm{E}-14$ & 1 & 50 & $8.88 \mathrm{E}-16$ & 0 & 50 \\
\hline $\mathrm{R}$ & $1.85 \mathrm{E}-11$ & 3 & 50 & $1.13 \mathrm{E}-12$ & 2 & 50 & $2.10 \mathrm{E}-09$ & 5 & 50 \\
\hline$S$ & $8.88 \mathrm{E}-16$ & 0 & 50 & $1.13 \mathrm{E}-12$ & 2 & 50 & $8.88 \mathrm{E}-16$ & 0 & 50 \\
\hline $\mathrm{T}$ & $8.88 \mathrm{E}-16$ & 0 & 50 & $2.81 \mathrm{E}-06$ & 9 & 50 & $2.23 \mathrm{E}-10$ & 4 & 50 \\
\hline $\mathrm{U}$ & $8.88 \mathrm{E}-16$ & 0 & 50 & $8.88 \mathrm{E}-16$ & 0 & 50 & $8.88 \mathrm{E}-16$ & 0 & 50 \\
\hline $\mathrm{V}$ & $1.61 \mathrm{E}-01$ & 21 & 50 & $8.88 \mathrm{E}-16$ & 0 & 50 & $8.88 \mathrm{E}-16$ & 0 & 50 \\
\hline $\mathrm{W}$ & $1.00 \mathrm{E}+00$ & 38 & 50 & $1.00 \mathrm{E}+00$ & 48 & 50 & $1.00 \mathrm{E}+00$ & 47 & 50 \\
\hline $\mathrm{X}$ & $2.23 \mathrm{E}-10$ & 4 & 50 & $4.53 \mathrm{E}-14$ & 1 & 50 & $1.13 \mathrm{E}-12$ & 2 & 50 \\
\hline $\mathrm{Y}$ & $1.00 \mathrm{E}+00$ & 48 & 50 & $8.88 \mathrm{E}-16$ & 0 & 50 & $8.88 \mathrm{E}-16$ & 0 & 50 \\
\hline $\mathrm{Z}$ & $4.44 \mathrm{E}-01$ & 24 & 50 & $9.68 \mathrm{E}-01$ & 31 & 50 & $8.99 \mathrm{E}-01$ & 29 & 50 \\
\hline
\end{tabular}

Table 1. Results of statistical tests of persistence. The results comprise three different cases: (1) outgoing, (2) incoming, and (2) total calls test of persistence. The p-value corresponds to the one obtained with a sign test. $\mathrm{N}+$ correspond to the number of inter-individual dissimilarities lower than the intra-dissimilarity. $\mathrm{N}_{\text {comp }}$ corresponds to the number of comparisons assessed.

statistically for every older individuals, and (2) may depend on the nature of the direction of calls considered in the analysis (outgoing, incoming or total calls).

Thus, as such, the statistical method we propose in this paper can be beneficial for all studies involving the persistence assessment for telephone call activity. In fact, the current methods used for assessing persistence in literature are mainly inherited from both (1) the field of complex network sciences ${ }^{38}$ - with a physical approach known as social physics ${ }^{15}$ - and (2) the field of computational social sciences ${ }^{39,40}$. Such methods hence rely rather on (1) the use of simple mathematical estimators integrated within a sophisticated formalistic scientific approach proper to social physics, and (2) a careful work of observation and interpretation of results which is proper to the field of social sciences than on a proper statistically consistent approach ${ }^{41}$. For applications associated with the understanding human social behavior, the combination of approaches (1) and (2) permits to address complex scientific questions on an original and relevant way. In particular, in the studies related to the telephone activity, mixing together quantitative and more qualitative approaches permits to bring relevant results relying on both objective elements of observation and on a careful work of interpretation, as evinced in ${ }^{21,25,30}$ for instance. Following this train of thought, integrating such an approach into a broader analytical process which includes statistical validation permits to introduce a certain level of significance regarding both the objective and subjective elements proposed and discussed in the involved studies. In particular, such a statistical assessment permits to nuance the results' interpretations proposed by the researchers by producing statistically valid elements that reinforce, or invalidate, the investigated theories. Such an inductive reasoning approach, which can seem evident for researchers working in the field of statistics, may be of a strong importance for new hybrids fields of computational science, such as social physics and computational social sciences.

Furthermore, it is interesting to mention that the novel statistical approach we propose in this paper can be situated into a broader, legitimate, scientific approach. In particular, regarding the combination of statistics with the concept of inter-intra dissimilarities used in the present study and introduced by Saramäki et al. ${ }^{21}$, such an approach has a long history and still remains of a strong importance in the fields of statistics and data analysis. Already at the beginning of the $\mathrm{XX}^{\text {th }}$ century, biometricians proposed to consider the inter-intra approach for comparing the mean effects observed in samples of interest. For instance, this was the case of Fisher who introduced the total variance decomposition that permits to distinguish the intra and inter group variances ${ }^{42}$. 


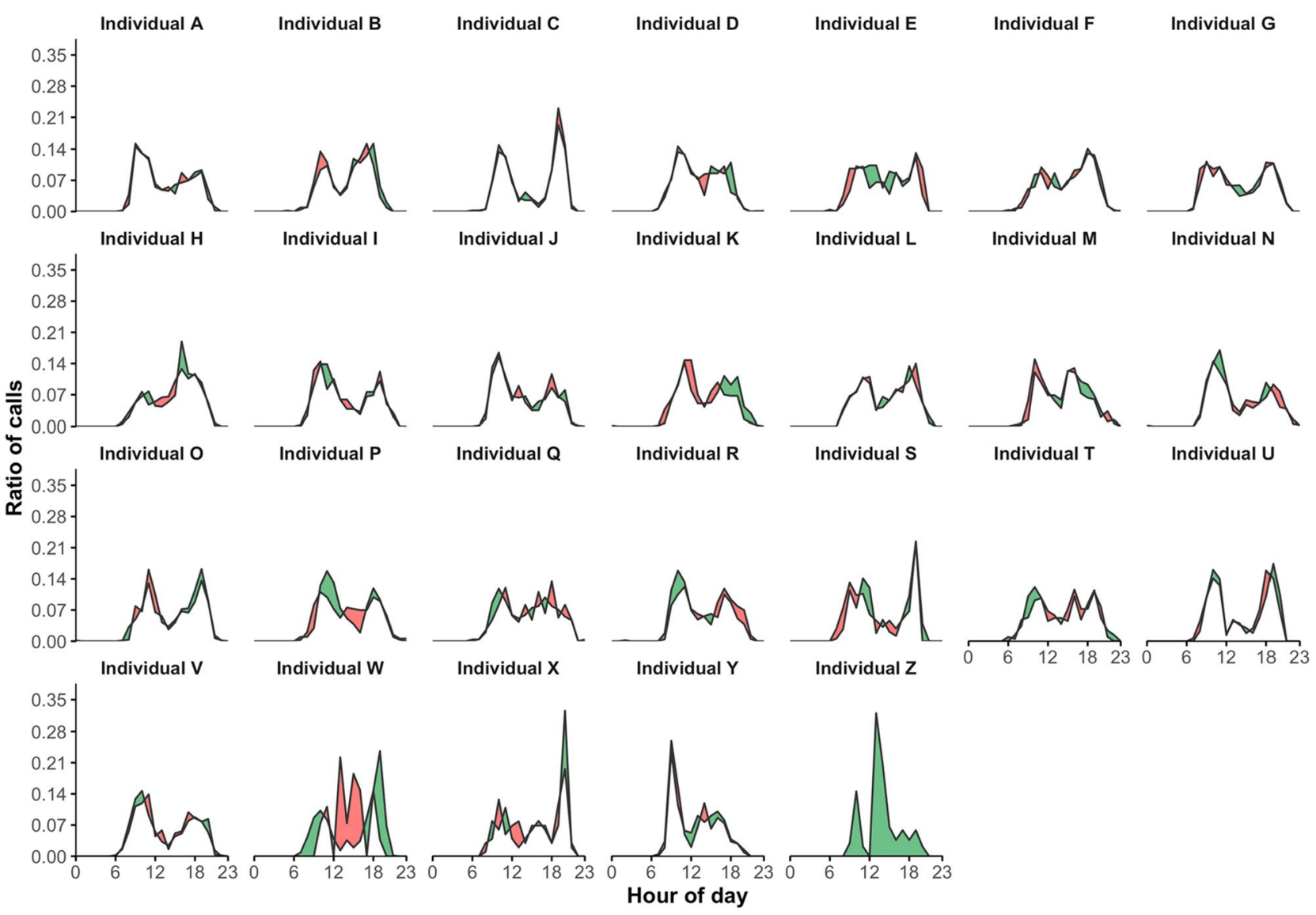

Figure 3. Comparison of two daily rhythms of incoming telephone call activity. Daily rhythms of incoming telephone call activity are calculated for the two successive time periods of 6 months each, $\mathrm{T}_{1}$ and $\mathrm{T}_{2}$. Their differences are illustrated by coloring the area between the two corresponding curves: (1) in green when the ratio of calls corresponding to $\mathrm{T}_{1}$ is higher than $\mathrm{T}_{2}$, and (2) in red for the opposite.

This approach is at the origin of the analysis of variance (ANOVA), which is, and remains to be, an essential approach for various domains of research requiring statistical evidences, such as the biomedical research sector for instance. Interestingly, this concept of inter-intra is also present at the core of recent data analysis approaches. For instance, in the field of statistical learning, unsupervised machine-learning methods usually rely on this concept for measuring the consistence of clusters obtained after the statistical analysis ${ }^{43}$. Thus, as such, the novel statistical approach we propose in this paper can benefit the complex and emerging approaches of persistence analysis used in the current literature by fitting this last one into a broader and coherent scientific framework.

Then, beyond these contextual elements underlying the framework of our approach, at least two elements of interest can be advanced for justifying the specific use of our statistical procedure in $m$ Health studies. First, it can inform with a certain level of significance about the personal nature of an individual' social behavior observed at telephone. For circadian rhythms specifically, there are yet evidences of singularity for the circadian periods of an individual ${ }^{1}$. Despite the precise and maintained entrainment of the circadian rhythms of activity on a 24-h clock, such an individual characteristic plays an essential role in the individual's life, and it is of strong importance in the field of chronobiology-and more broadly in personalized medicine-for addressing a patient's need adequately in time ${ }^{7}$. At the social level, the statistical procedure we propose can permit to address this essential point from a social and statistical view. Second, the objective and personal nature of data provided by modern phone technologies is presupposed in various fields of application related to personalized health ${ }^{13,36}$. Often, this assumption is used as an argument of interest regarding the use of such technologies in health (see recent reviews on the subject for instance ${ }^{13,36,44}$ ). As such, the statistical procedure we propose can permit to assess such a pre-notion of digital signature with regards to the daily social interactions occurring at telephone by assessing both their robustness and distinctiveness in time. For the clinical practice, such an assessment is of a strong importance before validating the personal nature of a patient's data generated by his phone device and, a fortiori, the personalized nature of the digital solution related to.

At this stage, however, it is important to recall that persistent analysis does not represent the only way to model consistent or inconsistent circadian rhythms from activity data. For instance, in another study ${ }^{45}$, LuqueFernandez et al. showed how an absence of circadian rhythms of a given physical activity can be modeled, by using alternative approaches than persistent analysis. In particular, these authors showed, in a totally different context, how specific behaviors such as labor can be modeled by fitting data about the number of labors at a given hour to a log-link trigonometric Poisson model. In particular, they investigated how such model can be used 


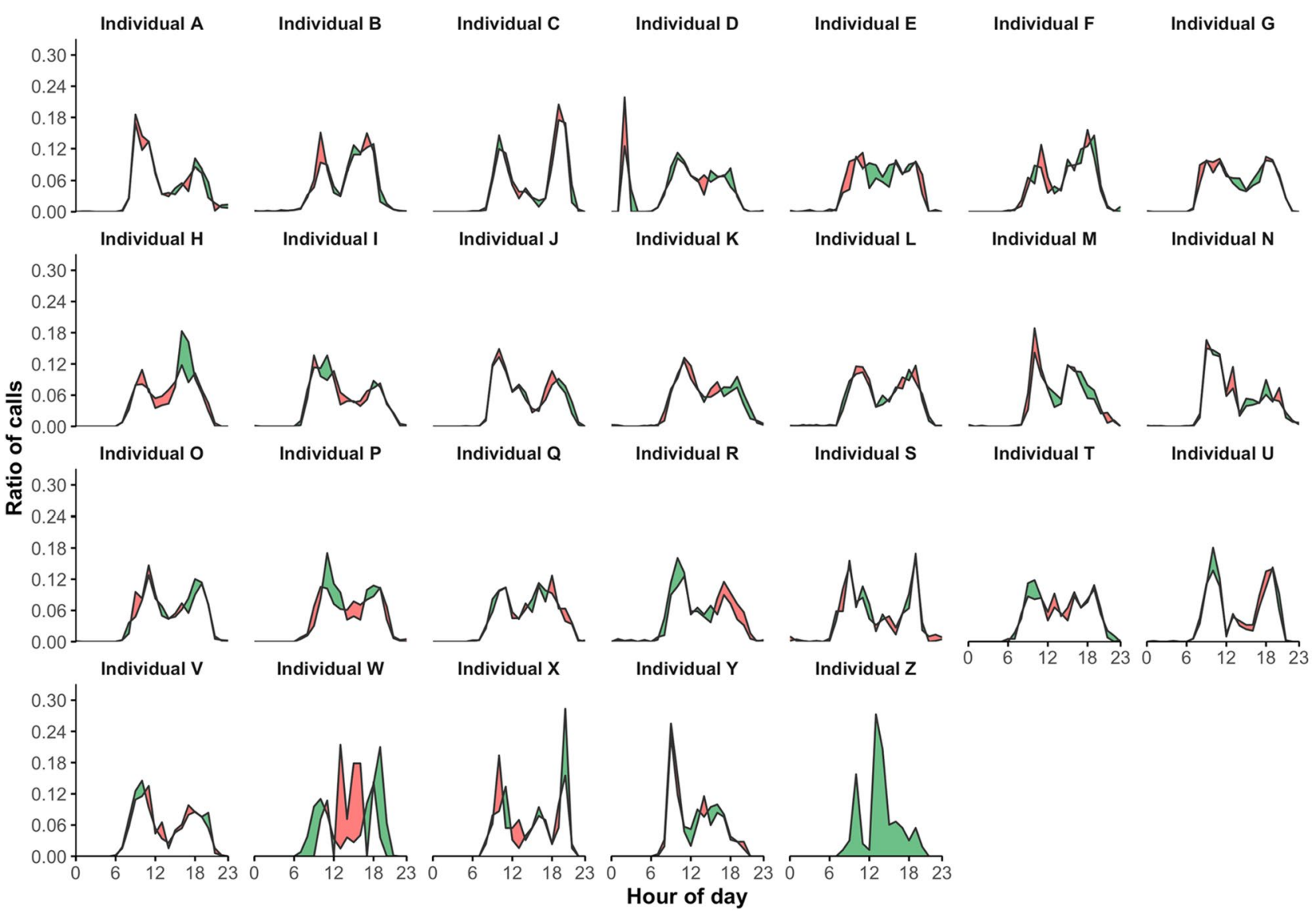

Figure 4. Comparison of two daily rhythms of total telephone call activity. Daily rhythms of total telephone call activity are calculated for the two successive time periods of 6 months each, $\mathrm{T}_{1}$ and $\mathrm{T}_{2}$. Their differences are illustrated by coloring the area between the two corresponding curves: (1) in green when the ratio of calls corresponding to $\mathrm{T}_{1}$ is higher than $\mathrm{T}_{2}$, and (2) in red for the opposite.

for detecting a damped sinusoidal behavior and how an observational Fourier analysis can permit to identify the presence of a stable periodicity. This approach was well adapted to the data observed in this study ${ }^{45}$ presenting an important variance. Here, the weak value of the variation coefficients (standard deviation/mean) of the data (equal for example to about $1 / 4$ for the reference- and self-distances used for comparing the daily phone activities) authorizes to use the average daily curves of phone activity as representative of a persistent highly not sinusoidal rhythm without estimating its Fourier fundamental and harmonic components. Taken together, these elements can underline the complementarity of persistence analysis with alternative ones, depending on the data analyzed and the nature of the activity to which they refer.

Thus, as such, the statistical procedure proposed in this study can be used for completing current methods of persistence analysis and for assessing the statistical validity of the existing results reported in the literature related to CDRs analysis. More importantly, it provides an opportunity to open up CDRs analysis to various domains of application that require an access to statistical significance, including health care monitoring. Finally, for health professionals, social information provided by the continuous, objective, unobtrusive analysis of persistent circadian rhythms of telephone activity can be used in complement of traditional punctual, subjective clinical questionnaires which require the active participation of the patient ${ }^{46,47}$. In the field of health care monitoring, such a use of phone technologies can permit to enhance the general framework around the analysis of the circadian rhythms of activity of an individual through time, which are of various natures. In particular, this enhancement is induced by the fact that there is no pure independency between the biological, physiological and social manifestations of the circadian rhythms ${ }^{4}$. On the contrary, these last ones are deeply entangled with each other. This complex interplay results in profound dependencies connecting the different levels of human life $^{4}$. In particular, as reported by Social Zeitgeber theory ${ }^{48}$, a biological alteration of the circadian rhythms can have adverse repercussions on social rhythms, and inversely. More importantly, such transverse alterations may act as a catalytic retroactive process that worsens the initial disruption which occurred in the individual's life. In this train of thought, there are evidences of significant associations between the occurrences of circadian rhythms' biological or social disruptions and various adverse situations including, but not limited to, mood disorders ${ }^{48}$, social jet-lag ${ }^{49}$, sleep disturbances ${ }^{50}$ or cognitive dysfunctions ${ }^{51}$, just for naming a few. Hence, in the field of health care monitoring, a deep comprehension of social manifestations of circadian rhythms can be of strong importance to properly analyze the circadian rhythms considering all their complexity and, then, for 
better managing a patient's health over time to prevent, when it possible, the occurrence or worsen of specific symptoms or diseases related to circadian rhythms alterations.

From the present work, a relevant perspective could consist of using the statistical analysis of persistence we propose for longitudinal studies in order to measure the robustness and distinctiveness of the circadian rhythms of telephone call activity of a population suffering from a specific illness or chronic disease such as bipolar disorder. Such an approach could permit to quantify the individuals' social rhythm regularity regarding specific disruptions. Such work could contribute to better analyze the role and manifestation of circadian rhythms of a social nature in health and to better understand their entanglement with biological and physical cues involved in the circadian rhythms of activity.

Received: 14 May 2020; Accepted: 19 October 2020

Published online: 08 December 2020

\section{References}

1. Ashkenazi, I. E., Reinberg, A., Bicakova-Rocher, A. \& Ticher, A. The genetic background of individual variations of circadianrhythm periods in healthy human adults. Am. J. Hum. Genet. 52, 1250-1259 (1993).

2. Mills, J. N. Human circadian rhythms. Physiol. Rev. 46, 128-171 (1966).

3. Ivanov, PCh., Hu, K., Hilton, M. F., Shea, S. A. \& Stanley, H. E. Endogenous circadian rhythm in human motor activity uncoupled from circadian influences on cardiac dynamics. Proc. Natl. Acad. Sci. 104, 20702-20707 (2007).

4. Ehlers, C. L., Frank, E. \& Kupfer, D. J. Social zeitgebers and biological rhythms: a unified approach to understanding the etiology of depression. Arch. Gen. Psychiatry 45, 948-952 (1988)

5. Burki, T. Nobel prize awarded for discoveries in circadian rhythm. Lancet 390, e25 (2017).

6. Seifalian, A. \& Hart, A. Circadian rhythms: will it revolutionise the management of diseases?. J. Lifestyle Med. 9, 1-11 (2019).

7. Kelly, R. M., Healy, U., Sreenan, S., McDermott, J. H. \& Coogan, A. N. Clocks in the clinic: circadian rhythms in health and disease. Postgrad. Med. J. 94, 653-658 (2018).

8. Cornelissen, G. \& Otsuka, K. Chronobiology of aging: a mini-review. Gerontology 63, 118-128 (2017).

9. Schwab, K. E. et al. Actigraphy to evaluate sleep in the intensive care unit. A systematic review. Ann. Am. Thorac. Soc. 15, 1075-1082 (2018).

10. Tazawa, Y. et al. Actigraphy for evaluation of mood disorders: a systematic review and meta-analysis. J. Affect. Disord. 253, 257-269 (2019).

11. Bardram, J. E. \& Matic, A. A decade of ubiquitous computing research in mental health. IEEE Pervasive Comput. 19, 62-72 (2020).

12. Blondel, V. D., Decuyper, A. \& Krings, G. A survey of results on mobile phone datasets analysis. EPJ Data Sci. 4, 1 (2015).

13. Sim, I. Mobile devices and health. N. Engl. J. Med. 381, 956-968 (2019).

14. The MQ Data Science group et al. How data science can advance mental health research. Nat. Hum. Behav. 3, 24-32 (2019).

15. Bhattacharya, K. \& Kaski, K. Social physics: uncovering human behaviour from communication. Adv. Phys. X 4, 1527723 (2019).

16. Eagle, N., Pentland, A. \& Lazer, D. Inferring friendship network structure by using mobile phone data. Proc. Natl. Acad. Sci. 106, 15274-15278 (2009).

17. Deville, P. et al. Dynamic population mapping using mobile phone data. Proc. Natl. Acad. Sci. 111, 15888-15893 (2014).

18. Finger, F. et al. Mobile phone data highlights the role of mass gatherings in the spreading of cholera outbreaks. Proc. Natl. Acad. Sci. 113, 6421-6426 (2016).

19. Pokhriyal, N. \& Jacques, D. C. Combining disparate data sources for improved poverty prediction and mapping. Proc. Natl. Acad. Sci. 114, E9783-E9792 (2017).

20. Vagni, G. \& Cornwell, B. Patterns of everyday activities across social contexts. Proc. Natl. Acad. Sci. 115, 6183-6188 (2018).

21. Saramäki, J. et al. Persistence of social signatures in human communication. Proc. Natl. Acad. Sci. USA 111, 942-947 (2014).

22. Park, P. S., Blumenstock, J. E. \& Macy, M. W. The strength of long-range ties in population-scale social networks. Science 362, 1410-1413 (2018).

23. Aubourg, T., Demongeot, J., Renard, F., Provost, H. \& Vuillerme, N. Association between social asymmetry and depression in older adults: a phone call detail records analysis. Sci. Rep. 9, 1 (2019).

24. Aubourg, T., Demongeot, J., Renard, F., Provost, H. \& Vuillerme, N. How to measure circadian rhythms of activity and their disruptions in humans using passive and unobtrusive capture of phone call activity. Stud. Health Technol. Inform. 264, 1631-1632 (2019).

25. Aledavood, T. et al. Daily rhythms in mobile telephone communication. PLoS ONE 10, e0138098 (2015).

26. Aledavood, T. et al. Channel-specific daily patterns in mobile phone communication. in Proceedings of ECCS 2014 (2016).

27. Aledavood, T., Lehmann, S. \& Saramäki, J. Digital daily cycles of individuals. Front. Phys. 3, 1 (2015).

28. Aubourg, T., Demongeot, J., Provost, H. \& Vuillerme, N. Circadian rhythms in the telephone calls of older adults: observational descriptive study. JMIR MHealth UHealth 8, e12452 (2020).

29. Stopczynski, A. et al. Measuring large-scale social networks with high resolution. PLoS ONE 9, e95978 (2014).

30. Alessandretti, L., Lehmann, S. \& Baronchelli, A. Understanding the interplay between social and spatial behaviour. EPJ Data Sci. 7, 1 (2018).

31. Saeb, S. et al. Mobile phone sensor correlates of depressive symptom severity in daily-life behavior: an exploratory study. J. Med. Internet Res. 17, e175 (2015).

32. Abdullah, S., Murnane, E. L. \& Matthews, M. \& Choudhury, T (Sensing, modeling, and maintaining biological rhythms. Mobile Health, Circadian Computing, 2017).

33. Lin, Y.-H., Wong, B.-Y., Pan, Y.-C., Chiu, Y.-C. \& Lee, Y.-H. Validation of the mobile app-recorded circadian rhythm by a digital footprint. JMIR MHealth UHealth 7, e13421 (2019).

34. Cho, C.-H. et al. Mood prediction of patients with mood disorders by machine learning using passive digital phenotypes based on the circadian rhythm: prospective observational cohort study. J. Med. Internet Res. 21, e11029 (2019).

35. Torous, J., Onnela, J.-P. \& Keshavan, M. New dimensions and new tools to realize the potential of RDoC: digital phenotyping via smartphones and connected devices. Transl. Psychiatry 7, e1053 (2017).

36. Bhugra, D. et al. The WPA-lancet psychiatry commission on the future of psychiatry. Lancet Psychiatry 4, 775-818 (2017).

37. Aubourg, T., Demongeot, J., Provost, H. \& Vuillerme, N. Exploitation of outgoing and incoming telephone calls in the context of circadian rhythms of social activity among elderly people: observational descriptive study. JMIR MHealth UHealth 8, e13535 (2020).

38. Holme, P. \& Saramäki, J. Temporal networks. Phys. Rep. 519, 97-125 (2012).

39. Lazer, D. et al. SOCIAL SCIENCE: computational social science. Science 323, 721-723 (2009).

40. Mann, A. Core concept: computational social science. Proc. Natl. Acad. Sci. 113, 468-470 (2016).

41. Bernard, H. R. The science in social science. Proc. Natl. Acad. Sci. 109, 20796-20799 (2012). 
42. Fisher, R. A., Bennett, J. H., Fisher, R. A., Fisher, R. A. \& Fisher, R. A. Statistical methods, experimental design, and scientific inference. (Oxford University Press, 1990).

43. Hastie, T. J., Friedman, J. \& Tibshirani, R. The elements of statistical learning data mining, inference, and prediction. (Springer, 2009).

44. Baumeister, H. \& Montag, C. Digital phenotyping and mobile sensing: new developments in psychoinformatics. (2019).

45. Luque-Fernandez, M. A. et al. Absence of circadian rhythms of preterm premature rupture of membranes and preterm placental abruption. Ann. Epidemiol. 24, 882-887 (2014).

46. Monk, T. K., Flaherty, J. F., Frank, E., Hoskinson, K. \& Kupfer, D. J. The social rhythm metric: an instrument to quantify the daily rhythms of life. J. Nerv. Ment. Dis. 178, 120-126 (1990).

47. Monk, T. H., Frank, E., Potts, J. M. \& Kupfer, D. J. A simple way to measure daily lifestyle regularity. J. Sleep Res. 11, 183-190 (2002).

48. Grandin, L. D., Alloy, L. B. \& Abramson, L. Y. The social zeitgeber theory, circadian rhythms, and mood disorders: review and evaluation. Clin. Psychol. Rev. 26, 679-694 (2006).

49. Malone, S. K., Mendoza, M. A. \& Patterson, F. Social jetlag, circadian disruption, and cardiometabolic disease risk. Sleep Health 1, 227-240 (2019)

50. Abbott, S. M., Knutson, K. L. \& Zee, P. C. Health implications of sleep and circadian rhythm research in 2017. Lancet Neurol. 17, $17-18$ (2018).

51. Wu, J. Q., Li, P., Gilbert, K. S., Hu, K. \& Cronin-Golomb, A. Circadian rest-activity rhythms predict cognitive function in early Parkinson's disease independently of sleep. Mov. Disord. Clin. Pract. 5, 614-619 (2018).

\section{Author contributions}

All authors contributed to this research. T.A., J.D. and N.V. contributed to conception and design of the study. N.V. contributed to data collection. T.A. organized the database. T.A. and J.D. performed the statistical analysis. T.A., J.D. and N.V. interpreted the data. T.A. wrote the first draft of the manuscript. T.A., J.D. and N.V. contributed to manuscript revision and gave approval for the final version prior to submission.

\section{Competing interests}

The authors declare no competing interests.

\section{Additional information}

Correspondence and requests for materials should be addressed to T.A.

Reprints and permissions information is available at www.nature.com/reprints.

Publisher's note Springer Nature remains neutral with regard to jurisdictional claims in published maps and institutional affiliations.

(i) Open Access This article is licensed under a Creative Commons Attribution 4.0 International License, which permits use, sharing, adaptation, distribution and reproduction in any medium or format, as long as you give appropriate credit to the original author(s) and the source, provide a link to the Creative Commons licence, and indicate if changes were made. The images or other third party material in this article are included in the article's Creative Commons licence, unless indicated otherwise in a credit line to the material. If material is not included in the article's Creative Commons licence and your intended use is not permitted by statutory regulation or exceeds the permitted use, you will need to obtain permission directly from the copyright holder. To view a copy of this licence, visit http://creativecommons.org/licenses/by/4.0/.

(C) The Author(s) 2020 